\title{
Evaluating the role of public health in implementation of genomics-related recommendations: a case study of hereditary cancers using the CDC Science Impact Framework
}

\author{
Ridgely Fisk Green, $\mathrm{PhD}, \mathrm{MMSc}^{1}$, Mary Ari, $\mathrm{PhD}^{3}$, Katherine Kolor, $\mathrm{PhD}, \mathrm{MS}^{2}$, W. David Dotson, $\mathrm{PhD}^{2}$, \\ Scott Bowen, $\mathrm{MPH}^{2}$, Nancy Habarta, $\mathrm{MPH}^{4}$, Juan L. Rodriguez, MPH, MS ${ }^{5}$, \\ Lisa C. Richardson, MD, MPH ${ }^{5}$ and Muin J. Khoury, MD, $\mathrm{PhD}^{2}$
}

Public health plays an important role in ensuring access to interventions that can prevent disease, including the implementation of evidence-based genomic recommendations. We used the Centers for Disease Control and Prevention (CDC) Science Impact Framework to trace the impact of public health activities and partnerships on the implementation of the 2009 Evaluation of Genomic Applications in Practice and Prevention (EGAPP) Lynch Syndrome screening recommendation and the 2005 and 2013 United States Preventive Services Task Force (USPSTF) BRCA1 and $B R C A 2$ testing recommendations. The EGAPP and USPSTF recommendations have each been cited by $>300$ peer-reviewed publications. CDC funds selected states to build capacity to integrate these recommendations into public health programs, through education, policy, surveillance, and partnerships. Most state cancer control plans include genomics-related goals, objectives, or strategies. Since the EGAPP recommendation, major public and private payers now provide coverage for Lynch Syndrome screening for all newly diagnosed colorectal cancers. National guidelines and initiatives, including Healthy People 2020, included similar recommendations and cited the EGAPP and USPSTF recommendations. However, disparities in implementation based on race, ethnicity, and rural residence remain challenges. Public health achievements in promoting the evidence-based use of genomics for the prevention of hereditary cancers can inform future applications of genomics in public health.

Genetics in Medicine (2019) 21:28-37; https://doi.org/10.1038/s41436018-0028-2

Keywords: evaluation; hereditary breast and ovarian cancer; Lynch syndrome; public health genomics; Science Impact Framework
Public health approaches to promoting health and preventing disease are population based, but frequently target population subgroups defined by characteristics such as race or ethnicity and rural or urban residential status. These subgroups might not represent the majority of those at risk, but often have a substantially greater risk than the general population. An emerging role for public health is to help find people at highest risk for hereditary cancer syndromes-a subgroup of the population at increased risk for cancer, especially at a younger age. Women with breast cancer 1 (BRCA1) and breast cancer 2 (BRCA2) pathogenic variants have a $69-72 \%$ risk of breast cancer and a $17-44 \%$ risk of ovarian cancer by age 80 , compared with lifetime breast and ovarian cancer risks of 12 and $1 \%$, respectively, for women in the general population. ${ }^{1,2}$ Women and men with Lynch syndrome (LS) have a 35 and $45 \%$ risk, respectively, of colorectal cancer by age 70 , compared with a $4.5 \%$ lifetime risk for the general population. $^{3,4}$ Women with LS have a $15-60 \%$ risk of endometrial cancer, compared with a $2.7 \%$ lifetime risk in the general population. ${ }^{4}$

BRCA pathogenic variants cause about $3 \%$ of breast cancers and $10 \%$ of ovarian cancers, ${ }^{5}$ while LS accounts for about $3 \%$ of colorectal cancers. ${ }^{3}$ Although most people diagnosed with breast, ovarian, or colorectal cancer do not have a BRCA or LS-related pathogenic variant, those carrying these pathogenic variants can find out about their cancer risk before any signs of disease, and take preventive measures early, when they are most likely to be effective. For women with $B R C A$ pathogenic variants, prophylactic mastectomy can reduce breast cancer risk by $85-100 \%$, and prophylactic oophorectomy can reduce ovarian cancer risk by $69-100 \%$ and breast cancer risk by $37-100 \%{ }^{5}$ Women with BRCA pathogenic variants can start mammography screenings earlier, as recommended by the United States Preventive Services Task Force (USPSTF), ${ }^{6}$

\footnotetext{
${ }^{1}$ Carter Consulting and Office of Public Health Genomics, Division of Public Health Information Dissemination, Center for Surveillance, Epidemiology, and Laboratory Services, Centers for Disease Control and Prevention, Atlanta, Georgia, USA; ${ }^{2}$ Office of Public Health Genomics, Division of Public Health Information Dissemination, Center for Surveillance, Epidemiology, and Laboratory Services, Centers for Disease Control and Prevention, Atlanta, Georgia, USA; ${ }^{3}$ Office of the Director, Office of the Associate Director for Science, Centers for Disease Control and Prevention, Atlanta, Georgia, USA; ${ }^{4}$ Division of Public Health Information Dissemination, Center for Surveillance, Epidemiology, and Laboratory Services, Centers for Disease Control and Prevention, Atlanta, Georgia, USA; ${ }^{5}$ Division of Cancer Prevention and Control, National Center for Chronic Disease Prevention and Health Promotion, Centers for Disease Control and Prevention, Atlanta, Georgia, USA. Correspondence: Ridgely Fisk Green (grf1@cdc.gov)
} 
along with magnetic resonance imaging, as recommended by the American Cancer Society. ${ }^{7}$ For individuals with LS, colorectal cancer screening reduces their lifetime colorectal cancer risk by about $62 \% .^{3}$

Evidence-based recommendations are an important first step in identifying and providing interventions to those at risk for disease, and translating these guidelines into public health practice is crucial for their implementation. The use of genomics in public health, especially in chronic disease prevention, is still emerging, and evaluations of ongoing efforts are important to provide the evidence base to show the impact of incorporating genomics into chronic disease prevention, to encourage and inform future efforts. Here, we use the Centers for Disease Control and Prevention (CDC) Science Impact Framework (SIF) ${ }^{8}$ to trace the influence of public health activities on the prevention of hereditary cancers. We focus on LS and BRCA-associated hereditary breast and ovarian cancer (HBOC), starting with the USPSTF 2005 and 2013 recommendation, "BRCA-related cancer: risk assessment, genetic counseling, and genetic testing" 2,9 and the 2009 Evaluation of Genomic Applications in Practice and Prevention (EGAPP) recommendation, "Genetic testing strategies in newly diagnosed individuals with colorectal cancer aimed at reducing morbidity and mortality from LS in relatives." 3 We selected the CDC SIF because it takes a broader approach to evaluation, measuring the impact of science beyond journal citations, and considers short-term indicators that support long-term impact, with an emphasis on contribution rather than attribution. The CDC $\mathrm{SIF}^{8}$ considers five spheres of influence: disseminating science, creating awareness, catalyzing action, effecting change, and shaping the future (Fig. 1).

\section{DISSEMINATING SCIENCE}

In 2009, the CDC-sponsored, independent EGAPP Working Group published a landmark evidence-based recommendation that genetic testing for LS should be offered to all individuals newly diagnosed with colorectal cancer, ${ }^{3}$ unlike previous guidelines, which recommended targeted screening based on age, Family health history, and other factors. Finding those with LS would allow their relatives to be tested for the same pathogenic variant and, if positive, to take steps to prevent cancer or find it early, including colonoscopies at a younger age. If everyone in the United States with newly diagnosed colorectal cancer were tested, about 4,000 people each year would be identified with LS. Testing the blood relatives of these 4,000 people could potentially identify multiple relatives in each family with LS who would benefit from evidence-based interventions.

To assess the evidence base before issuing the recommendation, the EGAPP working group conducted a systematic review on LS testing for all individuals with newly diagnosed colorectal cancer to reduce morbidity and mortality, updating an evidence report from the Agency for Healthcare Research and Quality. ${ }^{10}$ The supplementary review ${ }^{10}$ focused on clarifying the LS case definition, removing family health history assessment as a

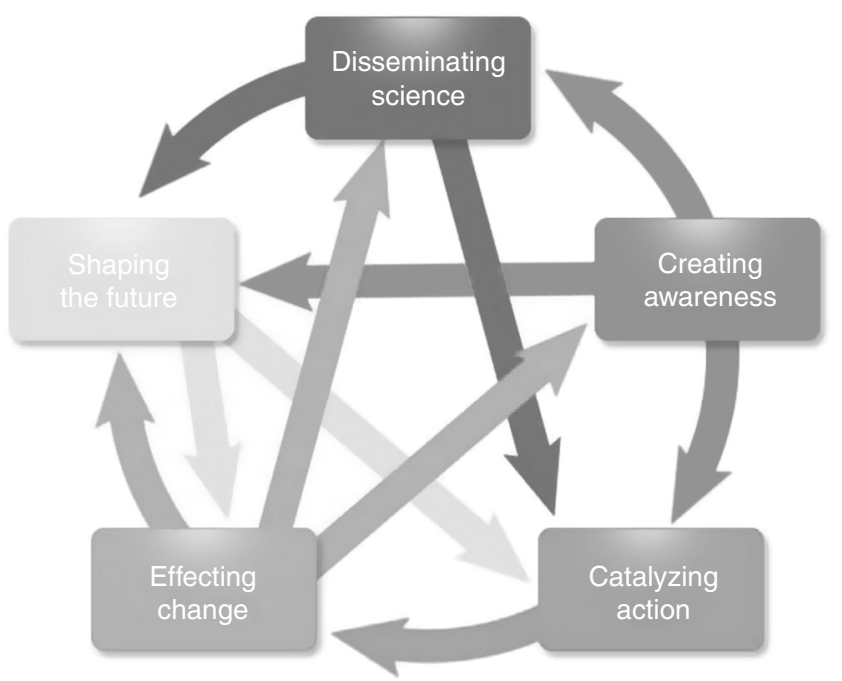

Fig. 1 Centers for Disease Control and Prevention Science Impact Framework. This framework ${ }^{8}$ illustrates the "historical tracing method", with five domains of Centers for Disease Control and Prevention scientific influence that define degrees of impact that may not be chronological. The degree of impact is not necessarily a progression; therefore, events captured may not be reflected at every domain. In addition, there may be loop-back at any point. Health outcomes are the ultimate goal, driven by the five domains of influence. Disseminating science can include publication of findings in peer-reviewed journals, presentation at conferences, or through other media channels. Creating awareness involves receiving recognition, and may include awards, general awareness, or acceptance of a concept or findings by the scientific community or policymakers, generating new discussion. Catalyzing action may include partnerships and collaborations, technology creation, congressional hearings or bills, or introduction in practice. Effecting change may include building public health capacity, legal or policy change, cultural, social, or behavioral change, or economic change. Shaping the future may include new hypotheses or strategies, implementation of new programs/initiatives, or quality improvement.

requirement for screening, determining the clinical validity of testing, identifying benefits and harms of testing for patients and their relatives, and providing a cost analysis.

The USPSTF published evidence-based recommendations in 2005 , revised in 2013, on the use of family health history to identify women at risk for $B R C A$ pathogenic variants (B rating). ${ }^{2,9}$ The recommendations focus on women with a family health history of cancer consistent with a BRCA pathogenic variant who have not had breast, ovarian, tubal, or peritoneal cancer themselves, and provide guidance targeted to primary care providers on referral for BRCA genetic counseling and testing. To identify women who should be referred for genetic counseling and, if indicated after counseling, BRCA genetic testing, the 2005 recommendation lists specific family health history criteria, while the 2013 revision states that primary care providers should screen women with one of several screening tools. An estimated 5\% of women in the United States-about 6 million women aged $\geq 18$ years-meet the 2005 USPSTF referral criteria (2014 US census estimates). ${ }^{11}$

\section{CREATING AWARENESS}

Over 340 peer-reviewed publications have cited the EGAPP LS screening recommendation, including cost-effectiveness 
studies supporting its universal screening approach in the United States, ${ }^{12}$ and studies describing the implementation of universal LS screening of newly diagnosed colorectal cancers ${ }^{13}$ (Supplementary Table S1 online). In a 2009 survey, 29\% of National Cancer Institute-designated comprehensive cancer centers, 16\% of American College of Surgeons-accredited community hospital comprehensive cancer programs, and $0 \%$ of community hospital cancer programs reported performing universal LS screening for all patients newly diagnosed with colorectal cancer. ${ }^{14}$ In a 2013 survey ${ }^{15}$ of pathology laboratories, about half reported screening all or nearly all colorectal cancers for LS, suggesting that LS screening rates had increased since 2009 (i.e., since the publication of the EGAPP recommendation). ${ }^{15}$

Over 400 peer-reviewed publications have cited the 2005 and/or 2013 USPSTF recommendations. These include studies on implementing breast cancer risk assessment for women without a personal history of breast cancer, either in primary care settings ${ }^{16}$ or among women receiving screening mammograms $^{17}$ (Supplementary Table S1 online). Also included are studies evaluating hereditary cancer risk assessment tools and protocols, ${ }^{18}$ assessing primary care clinicians' ability to determine hereditary cancer risk, ${ }^{19}$ identifying ways to improve cancer risk assessment and access to genetic services for those at-risk, ${ }^{20}$ and describing the prevalence of (and characteristics associated with) referrals, genetic counseling, and testing for $\mathrm{HBOC}^{21}$ (Supplementary Table S1 online).

To educate clinicians about the recommendations, CDC collaborated with Medscape on expert commentaries ${ }^{22}$ on LS and the EGAPP recommendation in 2011, and BRCA pathogenic variants and the USPSTF recommendation in 2014. CDC partnered with the Georgia, Michigan, and Oregon Departments of Health and the National Coalition for Health Professional Education in Genomics (now the Jackson Laboratory Clinical and Continuing Education Program) to create an online continuing medical education course on $\mathrm{HBOC},{ }^{23}$ with $>7,000$ sessions since its launch in February 2014 (D. Duquette, personal communication). The American Medical Association and the College of American Pathologists developed continuing medical education courses on LS. The 2016 CDC grand rounds, "Cancer and family health history: using genomics for prevention" and summary publication discussed public health approaches to hereditary cancers and focused on LS and HBOC, including the EGAPP and USPSTF recommendations. ${ }^{24}$ The grand rounds reached $>790$ participants, and the resulting publication has an Altmetric score of 58 as of September 2017, ranking in the top $5 \%$ of all research outputs scored by Altmetric.

CDC developed the Know:BRCA risk assessment tool, ${ }^{25}$ launched in 2014, to help women and their health-care providers assess their risk for $B R C A$ pathogenic variants. CDC launched the "Bring Your Brave" campaign ${ }^{26}$ in 2015 to increase young women's knowledge about breast health and risk factors for early-onset breast cancer, including $B R C A$ pathogenic variants.

\section{CATALYZING ACTION}

CDC funding helped establish selected state health departments' capacity to integrate $\mathrm{HBOC}$ into public health programs, starting in 2008 and continuing in 2011. The most recent funding in 2014 included LS and focused on education, policy, and surveillance. Trivers et $\mathrm{al}^{27}$ used CDC's SIF to evaluate funded states' $\mathrm{HBOC}$ activities.

\section{Educational activities}

Educational activities of CDC-funded state health departments include small media targeting providers and the public, online and in-person presentations and training, websites, publications, promotion of educational programs through provider incentives, creation of screening tools, development of genomics competencies and curriculum, technical assistance, national and state health observances, outreach events, and health education campaigns. For example, the Connecticut Department of Public Health developed an educational booklet for providers, Cancer Genomics Best Practices for Connecticut Healthcare Providers, and the Michigan Department of Health and Human Services (MDHHS) developed a handheld provider tool, the Cancer Family Health History Guide and a form and patient education booklet for providers to use to obtain written informed consent before presymptomatic or predictive genetic testing as mandated by Michigan law. Several states issued proclamations for LS Awareness Day on 22 March 2018, indicating that increasing LS awareness is a state priority. Michigan issued proclamations for HBOC Awareness Week from 25 September to 1 October 2016.

\section{Policy and systems change activities}

Policy and systems change activities conducted by CDCfunded states include developing cancer genomics program infrastructure, forming advisory committees, including genomics in state cancer plans, developing policy guidance documents for institutions and policymakers, working with state cancer registries to include data elements on cancer family health history and other genetic data, working with payers to promote coverage according to EGAPP and USPSTF recommendations, working with community clinics serving low-income populations to include family health history risk assessment, implementing a process in which laboratory reports on new colorectal cancer diagnoses are immediately forwarded to the local hospital cancer registrar and board-certified genetic counselor, encouraging compliance with the American College of Surgeons' "Commission on Cancer Genetics Standard: Risk Assessment and Genetic Counseling", and educating stakeholders about state licensure for genetic counselors.

We assessed state cancer plans currently available online for genomics terms similar to those used by Laufman et al.: ${ }^{28}$ gene, genetic, genomics, heredity, hereditary, heritability, family health history, DNA, high risk, risk assessment, and first-degree relative (Table 1). The majority of states $(71 \%$ (36/51), including Washington, DC; Table 1) include genomics-related goals, objectives, or strategies in their state 
Table 1 Genomics, HBOC, and LS in state cancer plans

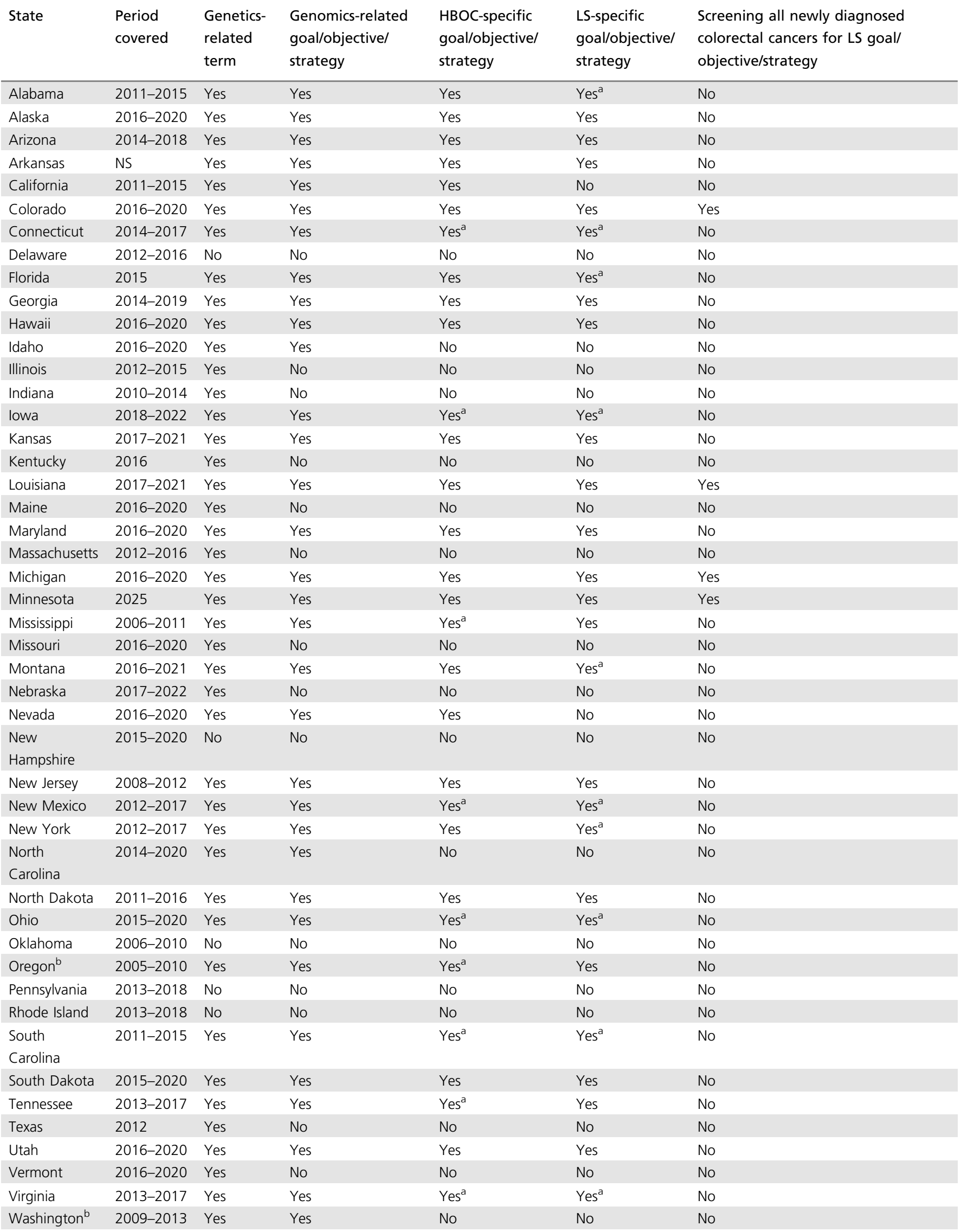


Table 1 continued

\begin{tabular}{|c|c|c|c|c|c|c|}
\hline State & $\begin{array}{l}\text { Period } \\
\text { covered }\end{array}$ & $\begin{array}{l}\text { Genetics- } \\
\text { related } \\
\text { term }\end{array}$ & $\begin{array}{l}\text { Genomics-related } \\
\text { goal/objective/ } \\
\text { strategy }\end{array}$ & $\begin{array}{l}\text { HBOC-specific } \\
\text { goal/objective/ } \\
\text { strategy }\end{array}$ & $\begin{array}{l}\text { LS-specific } \\
\text { goal/objective/ } \\
\text { strategy }\end{array}$ & $\begin{array}{l}\text { Screening all newly diagnosed } \\
\text { colorectal cancers for LS goal/ } \\
\text { objective/strategy }\end{array}$ \\
\hline West Virginia & 2016-2020 & Yes & Yes & Yes & Yes & Yes \\
\hline Wisconsin & $2016-2020$ & Yes & Yes & $Y_{e s}{ }^{a}$ & $Y_{e s}{ }^{a}$ & No \\
\hline
\end{tabular}

cancer control plans, and the number has continued to increase, even as cancer control plans have become more streamlined. (Laufman et al.'s 2012 study found that 64\% (32/ 50 ) of state cancer plans included genomics-related goals, strategies, or objectives. ${ }^{28}$ ) Most cancer control plans that are up-to-date $(72 \%(21 / 29))$ include genomics-related goals, objectives, or strategies. Five state cancer plans include goals, objectives, or strategies on LS screening of all newly diagnosed colorectal cancers. Nineteen state cancer control plans include goals, objectives, or strategies that address LS or family health history of colorectal cancer, 23 plans include goals, objectives, or strategies that address HBOC or BRCA testing, and an additional 10-11 include goals, objectives, or strategies that are relevant to HBOC and LS but use more general terms, such as hereditary cancer or family health history of cancer.

\section{Surveillance activities}

Surveillance activities include: surveys of providers, patients, and payers to assess knowledge, interest, and current practices regarding family health history, hereditary cancers, and genetic testing; the addition of questions on family health history of cancer and hereditary cancers to state surveys such as the Behavioral Risk Factor Surveillance System (BRFSS); hospital chart reviews to track the number of patients with newly diagnosed colorectal cancer screened for LS; and data collection through state-specific surveillance systems.

Using data from surveys, such as the BRFSS, states have been able to estimate the state prevalence of personal and family health history of breast, colorectal, and other cancers, assess awareness of and interest in genetic testing for hereditary cancer syndromes, and track state progress toward national goals and objectives and state cancer plan goals, objectives, or strategies. A study using data from the 2006-2009 Oregon BRFSS ${ }^{29}$ found that health-care providers were more likely to discuss cancer risk, screening for breast cancer, and health behavior changes with patients with a family health history of breast cancer, compared with those without a family health history of breast cancer. Women who discussed breast cancer screening with their providers were more likely to have mammograms than those who did not discuss it. Analyses using the Michigan BRFSS ${ }^{30}$ showed a twofold increase in the percentage of Michigan women with a significant family health history of breast and/or ovarian cancer (based on the 2005 USPSTF criteria) who received genetic counseling $(8.5-8.8 \%$ in $2012 ; 16.0 \%$ in 2015$)$, with about $10 \%$ of adult Michigan women meeting the 2005 USPSTF criteria for genetic counseling referral. Data from the 2010 Michigan BRFSS ${ }^{31}$ showed that a higher percentage of adults with a personal or family health history of colorectal cancer reported having a colon cancer screening than those who did not report a personal or family health history of colorectal cancer (80.4 vs. 65.3\%). A 2008 Oregon BRFSS study found that respondents with a family health history of colorectal cancer were more likely to report that their healthcare provider discussed colorectal cancer screening (odds ratio $=4.2(95 \%$ confidence interval $=2.4-7.4))$, that they had colorectal screening within the recommended time period (odds ratio $=2.2(95 \%$ confidence interval $=1.3-3.9)$ ), and that they made lifestyle changes to prevent colorectal cancer $($ odds ratio $=2.6(95 \%$ confidence interval $=1.7-4.0)){ }^{32}$

\section{Partnerships and other activities}

State cancer genetics programs have partnered with cancer registries, clinical facilities, health-care providers, health systems, public and private payers, policymakers, other state, regional, and federal programs, academic institutions, community organizations, advocacy groups, and industry. The LS Screening Network (LSSN), ${ }^{33}$ created in 2011, fosters collaboration and data sharing among institutions routinely screening newly diagnosed colorectal or endometrial cancers for LS. Thus far, 122 leading cancer institutions in 30 states have applied for LSSN membership, and 95 institutions and partners in 30 states are active members. Ninety-five percent of LSSN member institutions report that they used the EGAPP recommendation to justify or support their universal or routine LS screening of colorectal cancer cases (D. Duquette, personal communication). LSSN evolved from a 2010 CDC stakeholder meeting on universal colorectal cancer tumor screening for LS. ${ }^{34}$

One of the first approaches some states have used to implement hereditary cancer activities has been bidirectional reporting using cancer registries to identify individuals at increased risk for LS (those with colorectal cancer and endometrial cancer before 50 years of age) and HBOC (those 
with breast cancer before 50 years of age or ovarian cancer). These programs reported aggregate numbers of patients at increased risk to the reporting institution or provider and, in some cases, contacted the patient to inform them of their risk. While bidirectional reporting might not change clinical outcomes-in part due to the time elapsed between cancer diagnosis and reporting the risk-state health departments used it as an educational tool to promote compliance with the EGAPP, USPSTF, and other recommendations. ${ }^{35}$ The MDHHS cancer genomics program reported 10,340 colorectal cancers, 3,025 breast cancers in women $<50$ years of age, 1,985 people with multiple HBOC-related or LS-related primary cancers, 459 endometrial cancers in women $<50$ years of age, 127 ovarian cancers, and 147 male breast cancers. The Connecticut Department of Public Health reported $>3,700$ cancer cases for possible HBOC evaluation and received requests from $70 \%$ of participating hospitals for grand rounds presentations on prevention, early detection, and genetic counseling and testing for hereditary cancers. ${ }^{35}$ A 2009 project in Colorado ${ }^{36}$ reported hereditary colorectal cancer information on 575 cases to 412 health-care providers and 181 patients. HBOC bidirectional reporting programs are described further by Trivers et al. ${ }^{27}$

Using CDC-funded state activities as models, CDC developed the Public Health Genomics Toolkit ${ }^{37}$ to assist other state health departments in implementing the EGAPP and USPSTF recommendations. The Toolkit has been visited over 20,000 times since its launch and includes resources such as patient and provider fact sheets on LS and HBOC, summaries of the EGAPP and USPSTF recommendations, pamphlets and sample letters to help those with LS or BRCA pathogenic variants share information about their diagnoses with family members, and a slide set for states to use for educating providers and institutions about LS and HBOC, all of which can be customized to suit states' needs. To provide further access to public health genomics activities at the state level, CDC created the State Public Health Genomics Program Map, ${ }^{38}$ which provides state-by-state information on HBOC and LS activities, and has been visited $>65,000$ times.

\section{EFFECTING CHANGE}

For implementation of the USPSTF and EGAPP recommendations in the clinical setting, health insurance coverage for services related to $B R C A$ testing and universal LS screening may be necessary. The Patient Protection and Affordable Care Act requires many health plans to provide in-network coverage without cost sharing for preventive services with a USPSTF rating of " $\mathrm{A}$ " or " $\mathrm{B}$," which includes the $B R C A$ testing recommendation. ${ }^{39}$ A clarification ${ }^{40}$ in May 2015 stipulated that Patient Protection and Affordable Care Act coverage included women with a personal history of cancer, and the Centers for Medicare and Medicaid Services (CMS) Local Coverage Determinations on BRCA1 and BRCA2 Genetic Testing ${ }^{41}$ allow for regional coverage of $B R C A$ genetic counseling and testing for Medicare beneficiaries with personal histories of breast, ovarian, and other cancers that fit specific criteria for increased risk for a $B R C A$ pathogenic variant. Thus, coverage, depending on the source, can potentially be provided for individuals both with and without personal histories of BRCA-related cancers who meet certain criteria and have not previously undergone $B R C A$ genetic testing. An MDHHS study, ${ }^{42}$ using data before the Patient Protection and Affordable Care Act (2008-2012), found that insurance or out-of-pocket cost concerns were a substantial barrier for BRCA testing in women (with and without personal histories of breast or ovarian cancer) who had received $B R C A$ genetic counseling that indicated they were candidates for testing. A recent paper found a correlation between Patient Protection and Affordable Care Act coverage and increased $B R C A$ testing in women with a family health history of breast and/or ovarian cancer. ${ }^{43}$ Major private payers $^{44}$ and the $\mathrm{CMS}^{45}$ now provide coverage for LS screening, with some, including CMS, covering screening for all individuals diagnosed with colorectal cancer and citing the EGAPP recommendation.

The reach of the EGAPP and USPSTF recommendations and public health efforts to implement these recommendations has been magnified by the inclusion of similar recommendations in other guidelines and initiatives. Following the EGAPP recommendation, ten national and international recommendations have included universal LS screening. ${ }^{4,46-54}$ Recommendations from at least seven national and international organizations include strategies for identification of women at risk for BRCA pathogenic variants and cite the USPSTF recommendation. ${ }^{55-59}$

$B R C A$ testing and LS screening were included in national initiatives aimed at improving health. The Healthy People 2020 genomics objectives, ${ }^{60}$ which cite the USPSTF and EGAPP recommendations, are to "increase the proportion of women with a family health history of breast and/or ovarian cancer who receive genetic counseling" and "increase the proportion of persons with newly diagnosed colorectal cancer who receive genetic testing to identify LS (or familial colorectal cancer syndromes)." The 2016 National Cancer Institute Cancer Moonshot Blue Ribbon Panel Report ${ }^{61}$ recommended an LS demonstration project, which includes LS screening of all new colorectal cancers in the United States, and cited the EGAPP recommendation. The report included an $\mathrm{HBOC}$ demonstration project focused on genetic testing of men with breast cancer, women $<50$ years of age with breast cancer, and women with ovarian cancer. While the USPSTF recommendation starts with unaffected women whose risk is identified through their family health history, it acknowledges that testing should ideally first be done in a family member who has had a BRCA-related cancer. Also, the demonstration project would extend testing to relatives of individuals who test positive for a pathogenic variant.

Recent studies indicate that LS screening of colorectal cancer patients is not yet universal. A population-based study on those diagnosed in 2011 in Louisiana ${ }^{62}$ found that only $23 \%$ of the 274 colorectal cancer patients aged $\leq 50$ years were screened for LS. However, studies on institutions 
implementing universal screening have seen higher rates. ${ }^{13}$ As one measure of efficacy of the work to increase LS screening of all colorectal cancers, LSSN member institutions have screened $>31,000$ colorectal cancer cases since 2008, and LSSN provides a forum for providers to discuss questions on cases and screening (D. Duquette, personal communication). LSSN recently received funding for its database, which will provide an opportunity to track LS screening nationally across its member institutions.

Recent studies have shown increases in BRCA testing rates, and cancer family health history has surpassed personal history of breast or ovarian cancer as the indication for testing. ${ }^{63}$ This is consistent with more women being identified and tested in accordance with the USPSTF guidelines.

Studies have shown disparities by race and ethnicity and rural and urban residential status in the identification and treatment of those with hereditary cancer syndromes, highlighting opportunities for public health approaches that address these disparities. Black breast cancer survivors are less likely than breast cancer survivors of other races to have HBOC genetic counseling or testing. ${ }^{64-66}$ The most commonly reported reason is that their health-care provider has not recommended genetic services, ${ }^{64,65}$ and health-care providers primarily serving minority populations are less likely to refer or order genetic testing for their patients. ${ }^{67}$ Black people with $B R C A$ pathogenic variants are less likely to tell their relatives about their pathogenic variant, and relatives are less likely to be tested for the pathogenic variant. ${ }^{68}$ Furthermore, black women with $B R C A$ pathogenic variants have lower rates of risk-reducing salpingo-oophorectomy than BRCA carriers of other races. ${ }^{65}$ Similarly for colorectal cancer, colonoscopy screening at ages 40-49 for first-degree relatives of those with colorectal cancer was lower among black people than white people. ${ }^{69}$ Hispanic women with earlyonset breast cancer are also less likely to undergo BRCA testing, compared with non-Hispanic white women. ${ }^{66}$

Disparities have also been observed for those living in rural areas. A recent study ${ }^{70}$ looking at women with employeesponsored insurance found that BRCA testing rates were lower in nonmetropolitan areas compared with metropolitan areas, although the differences decreased over the study period, especially in younger women. Women living in nonmetropolitan areas were also less likely to receive certain preventive interventions. Universal LS screening of colorectal cancers is less common in community hospitals, which tend to serve rural populations, compared with institutions with comprehensive cancer centers or programs, ${ }^{14}$ which tend to be located in more urban areas.

Efforts to increase risk assessment and genetic testing in populations with lower rates have shown success. For example, a study offering genetic counseling and testing for HBOC to women at a safety-net hospital in which $78 \%$ of patients were from racial and ethnic minority groups had high uptake rates for these services. ${ }^{71}$ Future public health efforts can impact disparities in the implementation of the EGAPP and USPSTF recommendations.

\section{SHAPING THE FUTURE}

Work by the public health community to promote HBOC risk assessment and universal LS screening has helped lead to new initiatives and programs. In 2015, the National Academies of Sciences, Engineering, and Medicine's Roundtable on Genomics and Precision Health formed the Genomics and Population Health Action Collaborative ${ }^{72}$ to identify opportunities for genomics to improve population health, prevent disease, and reduce health disparities, inform and engage stakeholders about implementation of genomics, and explore ways to integrate evidence-based genomic applications into population health programs at the health-care-public-health interface. The Genomics and Population Health Action Collaborative focused on LS and HBOC and included evaluation and implementation working groups in the first year. Its evaluation working group finalized its work with the completion of a white paper $^{72}$ on "Building the Evidence Base for Genomics in Public Health: Implications for Decision Making, Public Policy, and Population Health Planning." To address issues specific to health disparities in the implementation of hereditary cancer risk assessment and prevention, the implementation working group created a subgroup focused on health disparities. In 2017, working groups were formed on cascade and population-based screening to address how to broaden the identification of individuals with LS and BRCA pathogenic variants beyond screening those who already have cancer, using either a cascade screening approach, in which family members of individuals identified with LS or BRCA pathogenic variants are offered genetic counseling and testing for the pathogenic variant that their relative has, or a population-based screening approach to identify those at risk independent of their family health history.

Some academic medical centers and health systems have already launched cascade screening programs for LS and $B R C A$ pathogenic variants, and some state health departments have worked on defining the role of public health in cascade screening. However, further work is needed, including clinician and patient education about the importance and process of cascade screening. Using data reported by Myriad and data from the state BRCA Clinical Network surveillance system, the Michigan Department of Community Health showed that single-site testing, used in cascade screening, increased by only $12.1 \%$ from 2008 to 2011, compared with increases of $72.2 \%$ in sequencing and $370.9 \%$ in rearrangement testing (D. Duquette, personal communication). MDHHS survey data showed that individuals identified with a $B R C A$ pathogenic variant and those who tested negative in a family with a known $B R C A$ pathogenic variant shared their $B R C A$ testing results with an average of 11.7 and 9.2 relatives, respectively. However, only 2.2 and 1.1 of their relatives had subsequent BRCA testing (D. Duquette, personal communication). Health disparities in cascade screening have been observed. Among those receiving HBOC genetic counseling, black patients present with a known familial pathogenic variant at lower rates than white patients (3.3 vs. $13.4 \%)$, thus showing lower rates of cascade screening (D. Duquette, personal communication). 
The National Cancer Institute convened a 2017 workshop ${ }^{73}$ on "Approaches to Blue Ribbon Panel recommendations: the case of LS" to review the Blue Ribbon Panel recommendations and discuss health-care delivery, knowledge gaps, and resources needed for implementation. The workshop helped lead to 2018 National Cancer Institute funding for implementation research on hereditary cancers, including LS and HBOC. ${ }^{74}$

\section{SUMMARY}

We used CDC's SIF to document the trajectory and influence of public health activities to implement genomics, starting with the EGAPP and USPSTF recommendations. The SIF is beneficial for examining the effects of public health activities to translate recommendations, which are broad and often difficult to delineate and measure on a causal pathway. A single product rarely produces impact in isolation, but rather produces effects in combination with other contributions. Effects may not follow chronologically. The SIF accounts for all of this. One challenge in using the SIF is establishing links between events and outcomes without a set protocol or database to search, especially retrospectively when opportunities to collect further data may not be available. Also, the SIF lacks measures of magnitude or quantitation of effect size.

Our evaluation shows how cancer prevention programs have translated clinical evidence-based recommendations for the identification of individuals at risk for hereditary cancer syndromes into activities for public health practice, with the long-term goal of improving health. For example, MDHHS worked to raise awareness and establish systems to improve HBOC screening and showed a twofold increase in genetic counseling for women with a family health history consistent with HBOC, using BRFSS data. The inclusion of LS- and HBOC-related goals, objectives, and strategies in state cancer plans and national initiatives, such as Healthy People 2020 and the Cancer Moonshot, can provide the impetus for state health departments to implement hereditary cancer activities. CDC funds the central state-based cancer registries, which could provide further opportunities for collaborating at the state level to move these agendas forward. Evaluating activities of CDC-funded states is important to increase the evidence base for future efforts by state health departments and others.

The continued role of public health is crucial to ensure that health disparities in the identification and treatment of hereditary cancer syndromes are addressed. These disparities have been recognized in some cases, but work is needed to effect change. The Genomics and Population Health Action Collaborative health disparities working group focuses on identifying and addressing these disparities, and the Cancer Moonshot funding includes the development of "optimum strategies for reaching diverse communities such as rural, racial/ethnic minorities and low-socioeconomic groups." Evaluations of this work will be needed to see whether it results in changes in health disparities in hereditary cancer risk assessment and prevention.

Comparing the implementation of the USPSTF and EGAPP recommendations is informative. HBOC-related recommendations and federal funding were available earlier than for LS. Clinicians responsible for carrying out the recommendations differ: primary care providers for USPSTF and pathologists for EGAPP, requiring different target audiences for provider education. Patients participate directly in HBOC screening by sharing family health history information, unlike with LS tumor tissue testing. Tracking LS screening using available databases is challenging: the biochemical test used for most LS screening has multiple applications, so its use does not necessarily indicate LS screening. However, the LSSN database will address this gap. The success of the Family-health-history-based USPSTF recommendation might impede progress of the EGAPP recommendation, as providers performing LS screening might assume that family health history information is required, highlighting the importance of clear public health messaging that all colorectal cancers should be screened.

Public health efforts to implement hereditary cancer prevention activities can serve as a model for future integration of genomics into public health approaches to chronic disease prevention, such as integration of familial hypercholesterolemia into heart disease prevention activities. To help those in the public health and clinical arenas determine which genomic applications are ready for implementation, CDC created an evidence-based classification of genomic tests and family health history, the Tier Table Database. ${ }^{75}$ The Tier Table ranks genomic applications in three tiers according to the level of evidence supporting their use, with tier 1 applications having a synthesized evidence base supporting implementation in practice. LS screening of all newly diagnosed colorectal cancers, based on the EGAPP recommendation, and the use of $\mathrm{HBOC}$ family health history for risk prediction for $B R C A$ genetic counseling referral, based on the USPSTF recommendation, are two of $>40$ tier 1 tests. CDC works to promote implementation of tier 1 genomic applications, and public health efforts to translate the EGAPP and USPSTF recommendations into public health activities can inform this work.

\section{ELECTRONIC SUPPLEMENTARY MATERIAL}

Supplementary material is linked to the online version of the paper at https://doi.org/10.1038/s41436-018-0028-2

\section{DISCLOSURE}

The authors declare no conflicts of interest.

\section{REFERENCES}

1. Kuchenbaecker KB, Hopper JL, Barnes DR, et al. Risks of breast, ovarian, and contralateral breast cancer for $B R C A 1$ and $B R C A 2$ mutation carriers. JAMA. 2017;317:2402-16

2. Moyer VA, United States Preventive Services Task Force. Risk assessment, genetic counseling, and genetic testing for BRCA-related cancer in women: U.S. Preventive Services Task Force recommendation statement. Ann Intern Med. 2014;160:271-81.

3. Evaluation of Genomic Applications in Practice and Prevention (EGAPP) Working Group. Recommendations from the EGAPP Working Group: genetic testing strategies in newly diagnosed individuals with colorectal cancer aimed at reducing morbidity and mortality from Lynch syndrome in relatives. Genet Med. 2009;11:35-41. 
4. National Comprehensive Cancer Network. NCCN Guidelines Version 1. Genetics/Familial High-Risk Assessment: Colon 2017. https://www.nccn. org/professionals/physician_gls/pdf/genetics_colon.pdf. Accessed 5 February 2018

5. Nelson HD, Pappas M, Zakher B, et al. Risk assessment, genetic counseling, and genetic testing for BRCA-related cancer in women: a systematic review to update the U.S. preventive services task force recommendation. Ann Intern Med. 2014:160:255-66.

6. Siu AL, United States Preventive Services Task Force Screening for breast cancer: U.S. Preventive Services Task Force recommendation statement. Ann Intern Med. 2016;164:279-96.

7. Saslow D, Boetes C, Burke W, et al. American Cancer Society guidelines for breast screening with MRI as an adjunct to mammography. Cancer $J$ Clin. 2007;57:75-89.

8. Centers for Disease Control and Prevention. CDC Science Making a Difference-Five Domains of Influence. https://www.cdc.gov/od/science/ impact/index.htm. Accessed 5 February 2018.

9. United States Preventive Services Task Force. Genetic risk assessment and BRCA mutation testing for breast and ovarian cancer susceptibility: recommendation statement. Ann Intern Med. 2005;143:355-61.

10. Palomaki GE, McClain MR, Melillo $S$, et al. EGAPP supplementary evidence review: DNA testing strategies aimed at reducing morbidity and mortality from Lynch syndrome. Genet Med. 2009;11:42-65.

11. Healthy People 2020 Progress Review: Cancer and Genomics. https:// www.healthypeople.gov/sites/default/files/HP2020_Cancer_and_ Genomics_Progress_Review_Slides.pdf. Accessed 5 February 2018.

12. Grosse SD, Palomaki GE, Mvundura M, et al. The cost-effectiveness of routine testing for Lynch syndrome in newly diagnosed patients with colorectal cancer in the United States: corrected estimates. Genet Med. 2015;17:510-1.

13. Cohen SA, Laurino $M$, Bowen DJ, et al. Initiation of universal tumor screening for Lynch syndrome in colorectal cancer patients as a model for the implementation of genetic information into clinical oncology practice. Cancer. 2016;122:393-401.

14. Beamer LC, Grant ML, Espenschied CR, et al. Reflex immunohistochemistry and microsatellite instability testing of colorectal tumors for lynch syndrome among US Cancer Programs and follow-up of abnormal results. J Clin Oncol. 2012;30:1058-63.

15. Volmar KE, Idowu MO, Souers RJ, et al. Molecular testing in anatomic pathology and adherence to guidelines: a College of American Pathologists Q-Probes study of 2230 testing events reported by 26 institutions. Arch Pathol Lab Med. 2015;139:1115-24.

16. Brannon Traxler L, Martin ML, et al. Implementing a screening tool for identifying patients at risk for hereditary breast and ovarian cancer: a statewide initiative. Ann Surg Oncol. 2014;21:3342-7.

17. Destounis $S$, Arieno A, Morgan R. Implementation of a risk assessment program in a breast-imaging community practice. Breast Cancer. 2016:23:273-8

18. Acheson LS, Zyzanski SJ, Stange KC, et al. Validation of a selfadministered, computerized tool for collecting and displaying the family health history of cancer. J Clin Oncol. 2006;24:5395-402.

19. Burke W, Culver J, Pinsky L, et al. Genetic assessment of breast cancer risk in primary care practice. Am J Med Genet A. 2009;149:349-56.

20. Cohen SA, Nixon DM. A collaborative approach to cancer risk assessment services using genetic counselor extenders in a multi-system community hospital. Breast Cancer Res Treat. 2016;159:527-34.

21. Bellcross CA, Leadbetter S, Alford SH, et al. Prevalence and healthcare actions of women in a large health system with a family health history meeting the 2005 USPSTF recommendation for BRCA genetic counseling referral. Cancer Epidemiol Biomark Prev. 2013;22:728-35.

22. Medscape CDC Expert Commentary. Genetic Testing for Lynch Syndrome in Colorectal Cancer. http://www.medscape.com/viewarticle/735522. Accessed 5 February 2018. Medscape CDC Expert Commentary. Genetics and BRCA in Primary Care. http://www.medscape.com/ viewarticle/832522. Accessed 5 February 2018.

23. The Jackson Laboratory. Hereditary Breast and Ovarian Cancer (HBOC). https://learn.education.jax.org/browse/hpe/cme/courses/hboc. Accessed 5 February 2018.

24. Rodriguez JL, Thomas CC, Massetti GM, et al. CDC grand rounds: family health history and genomics as tools for cancer prevention and control. MMWR Morb Mortal Wkly Rep. 2016;65:1291-4.

25. Centers for Disease Control and Prevention Know:BRCA Tool. https:// www.cdc.gov/cancer/breast/young_women/knowbrca.htm. Accessed 5 February 2018.
26. Centers for Disease Control and Prevention. Bring Your Brave Campaign https://www.cdc.gov/cancer/breast/young_women/bringyourbrave/index. htm. Accessed 5 February 2018.

27. Trivers KF, Rodriguez JL, Cox SL, et al. The activities and impact of state programs to address hereditary breast and ovarian cancer, 2011-2014. Healthcare (Basel). 2015;3:948-63.

28. Laufman JD, Duquette D, Trepanier A. Evaluation of state comprehensive cancer control plans for genomics content. Prev Chronic Dis. 2012;9: E176.

29. Zlot Al, Cox SL, Silvey K, et al. The effect of chronic disease family health history on healthcare provider practice and patient behavior among Oregonians. Public Health Genom. 2012;15:189-200.

30. Fussman C, Schrager J, Duquette, D. Breast and Ovarian Cancer Personal/ Family Health History and Genetic Counseling Utilization Among Michigan Women. Michigan BRFSS Surveillance Brief. Vol. 10, No. 3. Lansing, Ml: Michigan Department of Health and Human Services, Lifecourse Epidemiology and Genomics Division, December 2016

31. Fussman C, Hager P, Searls ML. Colorectal Cancer Screening among Michigan Adults Aged 50 Years and Older. Michigan BRFSS Surveillance Brief. Vol. 8, No. 5. Lansing, MI: Michigan Department of Community Health, Lifecourse Epidemiology and Genomics Division, Chronic Disease Epidemiology Section, November 2014.

32. Zlot Al, Silvey K, Newell N, et al. Family health history of colorectal cancer: clinicians' preventive recommendations and patient behavior. Prev Chronic Dis. 2012:9:E21.

33. Mange S, Bellcross C, Cragun D, et al. Creation of a network to promote universal screening for Lynch syndrome: the Lynch Syndrome Screening Network. J Genet Couns. 2015:24:421-7.

34. Bellcross CA, Bedrosian SR, Daniels E, et al. Implementing screening for Lynch syndrome among patients with newly diagnosed colorectal cancer: summary of a public health/clinical collaborative meeting. Genet Med. 2012:14:152-62.

35. Senier $L$, Lee $R$, Nicoll $L$. The strategic defense of physician autonomy: state public health agencies as countervailing powers. Soc Sci Med. 2017:186:113-21

36. Lowery JT, Axell L, Vu K, et al. A novel approach to increase awareness about hereditary colon cancer using a state cancer registry. Genet Med. 2010;12:721-5.

37. Centers for Disease Control and Prevention. Tier 1 Genomic Applications Toolkit for Public Health Departments. https://www.cdc.gov/genomics/ implementation/toolkit/index.htm. Accessed 5 February 2018.

38. Centers for Disease Control and Prevention. State Public Health Genomics Program Map. https://phgkb.cdc.gov/PHGKB/stateMapStartPage.action. Accessed 5 February 2018

39. H.R.3590-Patient Protection and Affordable Care Act. https://www. congress.gov/bill/111th-congress/house-bill/3590. Accessed 5 February 2018.

40. Centers for Medicare \& Medicaid Services. FAQs About Affordable Care Act Implementation (Part XXVI). https://www.cms.gov/CCIIO/Resources/ Fact-Sheets-and-FAQs/Downloads/aca_implementation_faqs26.pdf. Accessed 5 February 2018.

41. For example, Local Coverage Determination (LCD): MolDX: BRCA1 and BRCA2 Genetic Testing (L36082). https://www.cms.gov/medicarecoverage-database/details/lcd-details.aspx? LCDId $=36082 \&$ ver $=26 \&$ CoverageSelection $=$ Both\&ArticleType $=$ All\&PolicyType $=$ Final $\& s=$ All\&KeyWord=brca\&KeyWordLookUp=Title\&KeyWordSearchTy$p e=A n d \& b c=g A A A A C A A A A A A A A \% 3 d \% 3 d \&$. Accessed 5 February 2018 and Local Coverage Determination (LCD): MolDX: BRCA1 AND BRCA2 Genetic Testing (L36163). https://Www.cms.gov/medicarecoverage-database/details/lcd-details. aspx? LCDId $=36163 \&$ ver $=3 \&$ SearchType $=$ Advanced \&CoverageSelection $=$ Local\&PolicyType $=$ Both \&$s=\&$ AdvSearchName $=5 \% 7 c 2 \&$ KeyWord $=B R C A 1+$ and + BRCA2\& KeyWordLookUp $=$ Title\&KeyWordSearchType $=$ Exact\&kq $=$ true\&b$c=$ IAAAACAAAAAAAA\%3d\%3d\&, Accessed 5 February 2018.

42. Hayden S, Mange S, Duquette D, Petrucelli N, Raymond VM, Partners $B C N$. Large, prospective analysis of the reasons patients do not pursue BRCA genetic testing following genetic counseling. J Genet Couns. 2017 26:859-65.

43. Han X, Jemal A. Recent patterns in genetic testing for breast and ovarian cancer risk in the U.S. Am J Prev Med. 2017:53:504-7.

44. For example, BlueCross BlueShield of North Carolina Corporate Medical Policy: Genetic Testing for Colon Cancer. http://www.bcbsnc.com/assets/ services/public/pdfs/medicalpolicy/genetic_testing_ for_colon_cancer.pdf. Accessed February 5, 2018. BlueCross BlueShield 
of Rhode Island Medical Coverage Policy: Genetic Testing for Lynch Syndrome and Other Inherited Intestinal Polyposis Syndromes-PREAUTH. https://www.bcbsri.com/sites/default/files/polices/

GeneticTestingLynchSyndrome.pdf. Accessed February 5, 2018. HMSA Genetic Testing for Lynch Syndrome And Other Inherited Colon Cancer Syndromes. https://hmsa.com/portal/provider/MM.02.007_Genetic_ Testing_for_Lynch_Syndrome_and_Other_Inherited_Colon_

Cancer_Syndromes_042216.pdf. Accessēd February 5, 2018. Aetna: Genetic Testing. http://www.aetna.com/cpb/medical/data/100_199/ 0140.html. Accessed February 5, 2018. Cigna Medical Coverage Policy: Tumor Profiling, Gene Expression Assays and Molecular Diagnostic Testing for Hematology/Oncology Indications. https://cignaforhcp.cigna.com/public/ content/pdf/coveragePolicies/medical/mm

0520_coveragepositioncriteria_tumor_profiling.pdf. Accessed February 5, 2018. Cigna Medical Coverage Policy: Genetic Testing for Hereditary Cancer Susceptibility Syndromes. https://cignaforhcp.cigna.com/public/content/pdf/ coveragePolicies/medical/mm_0518_coveragepositioncriteria_

genetic_cancer_syndromes.pdf. Accessed February 5, 2018. Humana Medical Coverage Policy: Genetic Testing for Colorectal Cancer Susceptibility. http://apps.humana.com/tad/tad_new/Search.aspx? criteria $=$ Lynch \&searchtype $=$ freetext \&policyType $=$ both. $\quad$ Accessed February 5, 2018. Anthem Medical Policy: Genetic Testing for Colorectal Cancer Susceptibility. https://www.anthem.com/ medicalpolicies/policies/mp_pw_c166601.htm. Accessed February 5, 2018. United Healthcare Medical Policy: Genetic Testing. https://www. unitedhealthcareonline.com/ccmcontent/Providerll/UHC/ en-US/Assets/ProviderStaticFiles/ProviderStaticFilesPdf/Tools \%20and\% 20Resources/Policies\%20and\%20Protocols/Medical\%20Policies/Medical \%20Policies/Genetic_Testing.pdf. Accessed February 5, 2018.

45. For example, Local Coverage Determination (LCD): MolDX: Genetic Testing for LYNCH SYNDROME (L35349). https://Www.cms.gov/ medicare-coverage-database/details/lcd-details.aspx? LCDId= 353499\&ver=159\&CoverageSelection=Both9\&ArticleType=All\&PolicyType $=$ Final9\&s=All9\&KeyWord=lynch + syndrome9\&KeyWordLookUp= Title9\&KeyWordSearchType $=$ And $9 \& b=$ gAAAACAAAAAA9\&. Accessed 11 June 2018.

46. Balmana J, Balaguer F, Cervantes A, et al. Familial risk-colorectal cancer: ESMO Clinical Practice Guidelines. Ann Oncol. 2013;24:vi73-vi80.

47. Duffy MJ, Lamerz R, Haglund C, et al. Tumor markers in colorectal cancer, gastric cancer and gastrointestinal stromal cancers: European group on tumor markers 2014 guidelines update. Int J Cancer. 2014;134:2513-22.

48. Giardiello FM, Allen J, Axilbund JE, et al. Guidelines on genetic evaluation and management of Lynch syndrome: a consensus statement by the US Multi-Society Task Force on colorectal cancer. Am J Gastroenterol. 2014;109:1159-79.

49. Rubenstein JH, Enns R, Heidelbaugh J, et al. American Gastroenterological Association Institute Guideline on the Diagnosis and Management of Lynch Syndrome. Gastroenterology. 2015;149:777-82.

50. Sepulveda AR, Hamilton SR, Allegra CJ, et al. Molecular biomarkers for the evaluation of colorectal cancer: guideline from the American Society for Clinical Pathology, College of American Pathologists, Association for Molecular Pathology, and American Society of Clinical Oncology. J Mol Diagn 2017;19:187-225.

51. Stoffel EM, Mangu PB, Gruber SB, et al. Hereditary colorectal cancer syndromes: American Society of Clinical Oncology Clinical Practice Guideline endorsement of the familial risk-colorectal cancer: European Society for Medical Oncology Clinical Practice Guidelines. J Clin Oncol. 2015;33:209-17.

52. Syngal S, Brand RE, Church JM, et al. ACG clinical guideline: genetic testing and management of hereditary gastrointestinal cancer syndromes. Am J Gastroenterol. 2015;110:223-62.

53. Weissman SM, Burt R, Church J, et al. Identification of individuals at risk for Lynch syndrome using targeted evaluations and genetic testing: National Society of Genetic Counselors and the Collaborative Group of the Americas on Inherited Colorectal Cancer joint practice guideline. J Genet Couns. 2012;21:484-93.

54. National Institute for Health and Care Excellence. Molecular Testing Strategies for Lynch Syndrome in People with Colorectal Cancer. https:// www.nice.org.uk/guidance/dg27/chapter/1-Recommendations. Accessed 5 February 2018.

55. Singapore Cancer Network Cancer Genetics W. Singapore Cancer Network (SCAN) Guidelines for Referral for Genetic Evaluation of
Common Hereditary Cancer Syndromes. Ann Acad Med Singapore. 2015;44:492-510.

56. Berliner JL, Fay AM, Cummings SA, et al. NSGC practice guideline: risk assessment and genetic counseling for hereditary breast and ovarian cancer. J Genet Couns. 2013;22:155-63.

57. Hampel H, Bennett RL, Buchanan A, et al. A practice guideline from the American College of Medical Genetics and Genomics and the National Society of Genetic Counselors: referral indications for cancer predisposition assessment. Genet Med. 2015;17:70-87.

58. Wilson RD, Langlois S, SOGC Genetics Committee. Genetic considerations for a woman's annual gynaecological examination. J Obstet Gynaecol Can. 2012;34:276-84.

59. National Comprehensive Cancer Network. NCCN Guidelines Version 2. 2017 Genetics/Familial High-Risk Assessment: Breast and Ovarian. https:// www.nccn.org/professionals/physician_gls/pdf/genetics_screening.pdf. Accessed 5 February 2018.

60. Office of Disease Prevention and Health Promotion. Healthy People 2020 Genomics Objective. https://www.healthypeople.gov/2020/topicsobjectives/topic/genomics. Accessed 5 February 2018.

61. National Cancer Institute. Cancer Moonshot ${ }^{\mathrm{SM}}$ Blue Ribbon Panel. https:// www.cancer.gov/research/key-initiatives/moonshot-cancer-initiative/ blue-ribbon-panel. Accessed 5 February 2018.

62. Karlitz JJ, Hsieh MC, Liu Y, et al. Population-based Lynch syndrome screening by microsatellite instability in patients $\leq 50$ : prevalence, testing determinants, and result availability prior to colon surgery. Am J Gastroenterol. 2015;110:948-55.

63. Chen Z, Kolor K, Grosse SD, et al. Trends in utilization and costs of BRCA testing among women aged 18-64 years in the United States, 2003-2014. Genet Med. 2017;20:428-34.

64. McCarthy AM, Bristol M, Domchek SM, et al. Health care segregation, physician recommendation, and racial disparities in BRCA1/2 testing among women with breast cancer. J Clin Oncol. 2016;34:2610-8.

65. Cragun D, Weidner A, Lewis $C$, et al. Racial disparities in BRCA testing and cancer risk management across a population-based sample of young breast cancer survivors. Cancer. 2017;123:2497-505.

66. Levy DE, Byfield SD, Comstock CB, et al. Underutilization of BRCA1/2 testing to guide breast cancer treatment: black and Hispanic women particularly at risk. Genet Med. 2011;13:349-55.

67. Shields AE, Burke W, Levy DE. Differential use of available genetic tests among primary care physicians in the United States: results of a national survey. Genet Med. 2008;10:404-14.

68. Fehniger J, Lin F, Beattie MS, et al. Family communication of $B R C A 1 / 2$ results and family uptake of $B R C A 1 / 2$ testing in a diverse population of BRCA1/2 carriers. J Genet Couns. 2013;22:603-12.

69. Tsai $\mathrm{MH}$, Xirasagar $\mathrm{S}$, de Groen PC. Persisting racial disparities in colonoscopy screening of persons with a family health history of colorectal cancer. J Racial Ethn Health Disparities; e-pub ahead of print 15 August 2017.

70. Kolor K, Chen Z, Grosse SD, et al. BRCA genetic testing and receipt of preventive interventions among women aged 18-64 years with employer-sponsored health insurance in nonmetropolitan and metropolitan areas-United States, 2009-2014. MMWR Surveill Summ. 2017;66:1-11.

71. Komenaka IK, Nodora JN, Madlensky L, et al. Participation of low-income women in genetic cancer risk assessment and BRCA 1/2 testing: the experience of a safety-net institution. J Community Genet. 2016;7:177-83.

72. National Academies of Sciences, Engineering, and Medicine. Action Collaboratives: Genomics and Population Health-A Precision Health Activity. $\quad$ http://www.nationalacademies.org/hmd/Activities/Research/ GenomicBasedResearch/Innovation-Collaboratives/Genomics-andPopulation-Health.aspx. Accessed 5 February 2018.

73. National Cancer Institute. Approaches to Blue Ribbon Panel Recommendations: The Case of Lynch Syndrome. https://cancercontrol. cancer.gov/lynch-syndrome-workshop/. Accessed 5 February 2018.

74. National Institutes of Health, National Cancer Institute. Approaches to Identify and Care for Individuals with Inherited Cancer Syndromes (U01). RFA-CA-17-041. https://grants.nih.gov/grants/guide/rfa-files/RFA-CA-17041.html. Accessed 5 February 2018.

75. Dotson WD, Douglas MP, Kolor K, et al. Prioritizing genomic applications for action by level of evidence: a horizon-scanning method. Clin Pharmacol Ther. 2014;95:394-402. 